\title{
COVID-19-related Posterior Reversible Encephalopathy Syndrome in an Adult ICU Patient in Ireland
}

\author{
Shankar Lal ${ }^{1}$, Ehtesham Khan ${ }^{1}$, Dara Diviney ${ }^{1}$, Jubil Thomas ${ }^{1}$, Tidi Hassan ${ }^{2}$ and Ian Counihan ${ }^{2}$ \\ ${ }^{1}$ Department of Anaesthesiology, Our Lady of Lourdes Hospital, Ireland \\ ${ }^{2}$ Department of Respiratory Medicine, Our Lady of Lourdes Hospital, Ireland
}

\begin{abstract}
Radiologically confirmed, novel coronavirus disease 2019 (COVID-19)-related neurological manifestations are being reported with increasing frequency since the outbreak of the disease. We describe a 54-year-old male with COVID-19, who demonstrated radiological and clinical findings of posterior reversible encephalopathy syndrome(PRES). The proposed mechanism suggested for COVID-19-related PRES is a disease induced inflammatory storm, which causes endothelial injury, resulting in endothelialdysfunction (ED), interstitial fluid extravasation and cerebral edema.
\end{abstract}

Key Words: COVID-19, Encephalopathy, Neurological complications, PRES.

How to cite this article: Lal S, Khan E, Diviney D, Thomas J, Hassan T, Counihan I. COVID-19-related Posterior Reversible Encephalopathy Syndrome in an Adult ICU Patient in Ireland. J Coll Physicians Surg Pak 2021; 31(JCPSPCR):CR87-CR89.

\section{INTRODUCTION}

Severe acute respiratory syndrome coronavirus 2 (SARS-CoV2) causes novel corona virus disease 2019 (COVID-19). COVID19 results in severe acute respiratory syndrome in a substantial number of patients, with 5-10\% requiring intensive care unit (ICU) admission and mechanical ventilation. ${ }^{1}$ Patients with COVID-19 may present with fever, dry cough and fatigue. ${ }^{2}$ However, there are case reports of patients with COVID-19 who present with only neurological signs and symptoms. ${ }^{3}$ Retrospective retrieval of data from Wuhan, China (where the disease was first reported), showed neurological symptoms of the central and peripheral nervous system; these symptoms were observed in $36.4 \%$ of the hospitalised patients with COVID$19 .{ }^{4}$ We present a case of a 54 -year-old male, morbidly obese, with hypertension and type 2 diabetes mellitus, who developed neurological complications and was finally diagnosed as posterior reversible encephalopathy syndrome (PRES).

\section{CASE REPORT}

A 54-year-old, morbidly obese male, known hypertensive with Type 2 diabetes mellitus, presented to Emergency Room with a shorthistory of severe respiratory distressand persistenthypoxemia.

Correspondence to: Dr. Shankar Lal, Department of Anaesthesiology, Our Lady of Lourdes Hospital, Ireland E-mail: shankar.anaesthesia1@gmail.com

Received: August 04, 2020; Revised: February 20, 2021; Accepted: March 11, 2021

DOI: https://doi.org/10.29271/jcpsp.2021.JCPSPCR.CR87
A chest X-ray on admission was highly suggestive of COVID-19 infection; and although initially undetected, COVID-19 was detected on subsequent nasal and throat swabs. After initial treatment with conventional oxygen therapy and non-invasive ventilation, hypoxaemia persisted; thus the decision was made to transfer the patient to the ICU for endotracheal intubation and mechanical ventilation. Management included deep sedation, intermittent use of neuromuscular blocking agents (NMBA) and several sessions of proning as part of his ventilatory management. However, he was generally intolerant of proning. The patient received hydroxychloroquineand azithromycin as per the hospital guidelines at the time.

He developed blood pressure lability during his ICU stay with a maximum blood pressure of $180 / 90 \mathrm{mmHg}$ recorded in a 24 hour period. He was recommenced on his routine oral medication for blood pressure control via a nasogastric tube; but, due to poor absorption, evidenced by high gastric aspirates, he was commenced on an intravenous infusion of labetalol.

Progressive evidence of an acute kidney injury subsequently resulted in continuous renal replacement therapy (CRRT). His ICU course was complicated by superimposed bacterial lung infection with E. Coli, H. Influenza, and methicillin-susceptible staphylococcus aureus (MSSA) detected on sputum culture.

By day-14 of ICU admission, intravenous infusions(IVI) of propofol, morphine and midazolam had been weaned off, and there was no further use of NMBAs. The patient received quetiapine $25 \mathrm{mg}$ and citalopram $20 \mathrm{mg}$, following discontinuation of intravenous sedation. Due to persistently low Glasgow coma scores (GCS) and negative Richmond agitation-sedation scale scores (RASS), a CT brain was done to rule out a cause of his low GCS/RASS score and protracted neurological recovery on 
day-32 of ICU admission. The CT brain was reported as normal. After a multi-disciplinary discussion, a decision was made to proceed with bed-side percutaneous dilatation tracheostomy on the $34^{\text {th }}$ day in ICU. Following tracheostomy, his ventilatory parameters started to improve gradually, but there was no evidence of improvement in his GCS. The maximum GCS recorded was 12/15, one week after tracheostomy. Urine samples for toxicology were taken on week one and two after discontinuation of IV sedation infusions, which were positive for benzodiazepines and opioids on both occasions. He was in the polyuric phase with a persistently deranged renal profile with urea of $27.7 \mathrm{mg} / \mathrm{dl}$ and creatinine $112 \mathrm{umol} / \mathrm{l}$ with normal electrolytes and $\mathrm{pH}$.

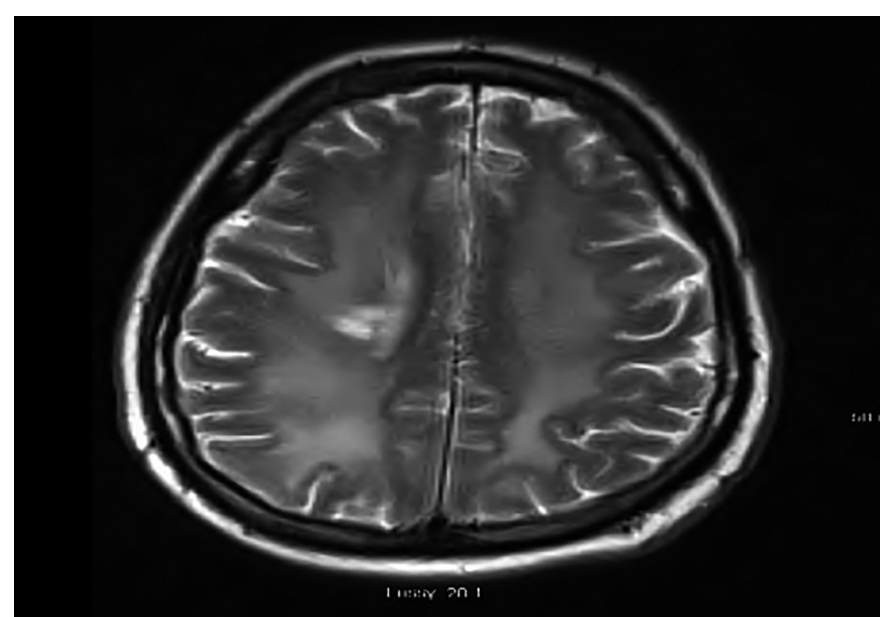

Figure 1: Axial T2 Flair showing hyperintensity, involving the right periventricular area. Deep white matter compatible with PRES.

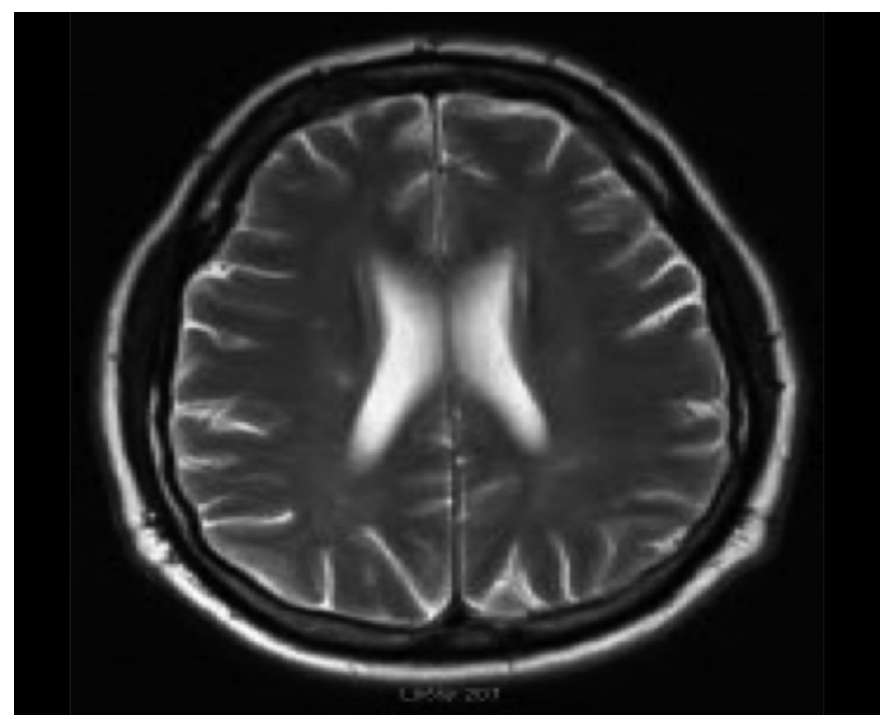

Figure 2:Axial T2 Flair showing complete resolution of hyperintensity of deep white matter, compatible with diagnosis of PRES.

On day- 53 of ICU admission, there was significant improvement in oxygenation, renal function, GCS and RASS; but on examination, it had become evident that the patient had motor weakness of both right and left (right side dominant) upper and lower limbs. Gradually, the right side improved to normal power, but residual left-sided weakness persisted. As there was no improvement in motor power on the left upper and lower limbs, an MRI brain without contrast was done that showed evidence of deep white matter intensity in the right periventricular area with the possibility of COVID-19-related encephalopathy (Figure 1).

A consult was generated for a neurology physician. The detailed neurological examination noted weak motor power of $1 / 5$ in the left upper limb and 2/5 in the left lower limb, while the motor power had returned to normal in the right upper and lower limbs. There was no evidence of dysphagia, dysphonia, decreased visual field, dis-coordination or abnormal reflexes on neurological examination. Thus, a differential diagnosis of COVID-19 encephalopathy was suggested.

To determine possible causes for encephalopathy leading to motor weakness, a detailed neurological screening was done. Cerebrospinal fluid (CSF) and serum were sent for viral and bacterial analysis, including PCR for COVID-19, human immunodeficiency virus (HIV), syphilis, and anti-galactomannan antibodies. CSF analysis showed high proteins with normal glucose and white blood cells with no growth in CSF culture. HSV1, HSV2, VZV and enteroviruses, HIV1 and HIV2 infection, and syphilis were not isolated, and an autoimmune workup, including paraneoplastic anti-bodies (Serum and CSF), neuronal antibodies (Serum and CSF), and monoclonal bands were negative.

Having outruled other causes of encephalopathy, clinical and radiological evidence on MRI brain suggested a diagnosis of COVID-19-related PRES.

On day-61, the patient had successfully weaned from mechanical ventilation; he was decannulated on day- 63 and was transferred to the stroke unit for further care. He continued to have intensive physiotherapy; and over a period of time, there was a gradual improvement in his left upper and lower limbs' motor power. By day-90, he had improved enough to walk independently and had no other evidence of neurological compromise.

A repeat MRI of brain, four weeks after discharge from the ICU, showed complete resolution of the deep right white matter signal abnormality, seen on the previous MRI. The patient was discharged home after 102 days in the hospital.

\section{DISCUSSION}

This is the first reported case of COVID-19-related PRES in the Republic of Ireland.

PRES is a rare syndrome, and its exact pathogenesis is not yet established. ${ }^{5}$ It may manifest due to cortical or subcortical vasogenicoedema in parietal and occipital regions of the brain resulting in headache, acute impairment in the level of consciousness, visual field defects and seizures. ${ }^{6}$ Blood pressure fluctuations, renal failure, severe sepsis, pregnancy-related hypertensive conditions, i.e. pre-eclampsia and eclampsia, and the use of immunosuppressive or cytotoxic drugs, have been associated with PRES. In this patient, PRES was most likely due to COVID-19-induced endothelial dysfunction (ED, which resulted in ischemia, inflammation and subsequent cerebral oedema in the right periventricular deep white matter. 
In COVID-19, there is evidence of direct viral binding to angiotensin-converting enzyme receptors, which may cause an increase in blood pressureand dysfunction ofcerebral autoregulation. ${ }^{7,8}$ Viral elements have been found in endothelial cells of different vascular beds suggesting the possible role of ED in the systemic toxicity induced by COVID-19. ${ }^{9}$

Other case reports are suggesting a relationship between COVID-19 and neurological symptoms manifested as acute stroke (6\%), impaired consciousness (15\%), and muscle injury $(19 \%) .{ }^{10}$ Patients with critical illness are at an increased risk of altered mental status in the setting of acute infection, prolonged pyrexia and acute kidney injury with protracted sedation.

We hypothesise, on the basis of clinical and radiological findings and the evidence of ED in COVID-19, that COVID-19 could have contributed to PRES in thispatient.

Patients with COVID-19 may be atrisk of PRES. Early and aggressive blood pressure control may decrease the risk of hypertensive encephalopathy in patients with COVID-19.

\section{PATIENT'S CONSET:}

The authors declared that patient's consent had been obtained.

\section{CONFLICT OF INTEREST:}

The authors declared no conflict of interest.

\section{AUTHORS'CONTRIBUTION:}

SL: Literature search, data retrieval and write-up.

$\mathrm{TH}$, IC, JT: Literature search and patient management.

DD: Literature search, data retrieval, supervision, and write-up.

EK: Final write-upand supervision.

\section{REFERENCES}

1. Wu Z, McGoogan JM. Characteristics of and important lessons from the coronavirus disease 2019 (COVID-19) outbreak in China: Summary of a report of 72314 cases from the Chinese center for disease control and prevention.
JAMA 2020; 323(13):1239-42. doi: 10.1001/jama.2020. 2648.

2. Outbreak in China: Summary of a report of 72314 cases from the chinesecentre for disease control and prevention. JAMA 2020.

3. Huang C, Wang Y, Li X, Ren L, Zhao J, Hu Y, et al. Clinical features of patients infected with 2019 novel coronavirusin Wuhan, China. Lancet 2020; 395(10223):497-506. doi: 10.1016/S0140-6736(20)30183-5.

4. Wang HY, Li XL, Yan ZR, Sun XP, Han J, Zhang BW. Potential neurological symptoms of COVID-19. Therapeutic $A d v$ Neurol Disord 2020; 13:1756286420917830. doi: 10.1177/ 175628642 dwwef0917830.

5. Mao L, Wang M, Chen S, He Q, Chang J, Hong C, et al. Neurological manifestations of hospitalized patients with COVID-19 in Wuhan, China: A retrospective case series study. SSRN Electronic J 2020;

6. Wu Y, Xu X, Chen Z, Duan J, Hashimoto K, Yang L, et al. Nervous system involvement afterinfection with COVID19 and other coronaviruses. Brain, Behavior Immun 2020; 87:18-22. doi: 10.1016/j.bbi.2020.03.031

7. Fischer M, Schmutzhard E. Posterior reversible encephalopathy syndrome. JNeurol 2017; 264(8):1608-16. doi: 10.1007/s00415-016-8377-8.

8. Baig AM, Khaleeq A, Ali U, Syeda H. Evidence of the COVID-19 virus targeting the cns: tissuedistribution, hostvirus interaction, and proposed neurotropic mechanisms. ACS Chemical Neuroscience 2020; 11(7): 995-98. doi: 10.1021/acschemneuro.0c00122.

9. Bogoch II, Watts A, Thomas-Bachli A, Huber C, Kraemer MUG, Khan K. Pneumonia of unknown aetiology in Wuhan, China: Potential for international spread via commercialair travel. J Travel Med 2020; 27(2): taaa008. doi:10.1093/ jtm/taaa008.

10. Mao L, Jin $\mathrm{H}$, Wang $M$, , Hu $Y, C$ Chen $S$, He Q, et al. Neurologic manifestations of hospitalized patients with coronavirus disease 2019 in Wuhan, China. Jama Neurol 2020; 77(6):683-90. doi: 10.1001/jamaneurol.2020.1127. 